\title{
An Evaluation of Location Management Procedures
}

\author{
Frank V. Baumann \& Ignas G. Niemegeers \\ Tele-Informatics and Open Systems Group - University of Twente \\ P.O. Box 217 - 7500 AE Enschede - The Netherlands \\ Tel.: +3153892819 - Fax: +3153333815 \\ E-mail: baumann@cs.utwente.nl
}

\begin{abstract}
This paper gives a comparative description of two scenarios for location management in a mobile telecommunications system. The first scenario uses fixed location and paging areas. Mobiles perform a location update as they enter a new location area. The second scenario uses a time-out based location updating scheme. Mobiles start their timer as they leave the paging area they are currently registered in. As the timer elapses, the mobile performs a location update. Both scenarios also differ in the way paging is performed. In the first scenario it is only necessary to page in the location area the mobile is currently registered in. In order to do this efficiently, the paging is done in a 2-step fashion: mobiles are paged first in the paging area in which they were registered in, and next in the entire location area they are registered in. In the second scenario the mobile is paged in multiple steps: first in the paging area it is registered in, next in a circle of paging areas surrounding that area, and so on, until the mobile is found, or the number of steps has reached a certain upper limit. Results comprise a quantitative and qualitative comparison of these scenarios, and guidelines for optimal application.
\end{abstract}

\section{Introduction}

It is expected that future mobile telecommunication networks will serve a very large number of subscribers. In order to keep the number of paging messages, needed to locate a mobile terminal (MT) in case of an incoming call, manageable, it is necessary to have a registration of the whereabouts of the MTs. In that case paging only has to be done in a limited area. Nevertheless, due to the very large number of subscribers, the number of messages related to location management procedures will still be huge.

Several papers describe approaches to diminish this large number of messages. In [1] some optimization techniques for location area (LA) partitioning are developed. Based on the flow of users between cells three approaches to determine the optimal LA partitioning are described. In [2] an approach is described which applies user profiles. These profiles are maintained on a per-user basis, and contain information about the areas in which the user most frequently roams. The previous approaches are applied and improved in [3], in which a large city with a lot of mobile subscribers is simulated, testing several alternatives for LA planning and intelligent paging strategies. In [4] a more analytical approach to the problem is pursued. A simple formula is applied which relates the user traffic flow out of an area to the size of the area, the density and average speed of users in that area, and the border length of the area. As an example the optimal LA size is calculated using this formula. Finally, in [5] a method is introduced which solves a related problem: the fact that all messages related to location up-

$\dagger$. The work on which this paper is based, forms a part of the RACE II MONET project, partly funded by the EEC, in which the Author is working. However, the views expressed are those of the Author and do not necessarily represent those of the project as a whole. dating are concentrated in the particular cells located at the LA periphery. The solution described here is a multi-layer location updating method.

The solutions mentioned above all have in common that when a MT crosses a LA border, a forced location update will be performed. This is not an optimal solution: for moving MTs which hardly ever receive incoming calls, a lot of unnecessary location updating needs to be done. The scenario proposed in this paper refrains from the principle of forced location updates, and introduces an alternative trigger for the location updating procedure. This concept introduces the need for paging of MTs outside their registered LA.

The work in this paper was done within the RACE II MONET project. This project deals with the design of a mobile telecommunications network for the near future, called Universal Mobile Telecommunications Network (UMTS). The study described here consists of a comparison between two scenarios for location management. The first scenario is a general fixed LA scenario, described in the RACE 1043 project [8][9]. The new scenario uses a timed location update scheme. Goal of this study is to give a quantitative comparison between these alternatives. The figures resulting should not be seen as absolute figures, but can be used to give insight in the advantages of one scenario over the other.

The performance parameters studied are:

- the terminal paging delay,

- the access rate on the UMTS distributed data base (DDB) due to location updates,

- the paging rate per paging area.

The approach is as follows. In Sections 2 and 3 a detailed description of the scenarios is given. Section 4 describes the UMTS scenario used as input for the analysis, which is based on previous mobility [10] and traffic [11] modelling work within the MONET project. In Section 5 an analytical model is developed for both scenarios, which generates the required network performance parameters, such as the generated amount of signalling for location updating and paging. The results of the evaluation are given in Section 6. Finally, Section 7 provides conclusions and identifies future research subjects.

\section{Fixed Location Area Scenario}

The most simple set of location management procedures, as described in [9], uses fixed LAs, each containing several cells. MTs perform a location update as they enter a new LA. In order to have a further reduction of the number of paging messages, LAs are subdivided into paging areas (PAs). A MT will first be paged in the PA where it had the last contact with the fixed network (which is called the registered PA). If it is not found, it will next be paged simultaneously in the remaining PAs of the LA. 


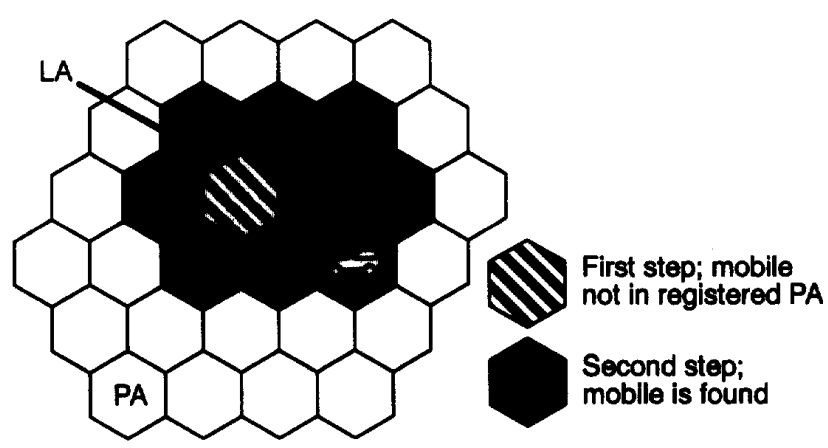

Figure 1: Example of two-step paging procedure

This system of location management leads to a number of location updates (thus updates in the DDB) per MT more or less proportional with the speed of that MT. The number of database queries and the amount of paging is proportional to the rate of calls destined to MTs. The exact amount of paging needed for the location of a particular MT depends on the fact whether this MT is still in its registered $\mathrm{PA}$.

The characteristics of the paging scenario are shown in Table 1. Two delay parameters are defined: $d_{\text {found }}$ which refers to successful paging; it is the mean delay between the broadcasting of a paging message and the receipt of the answer from the MT, and $d_{\text {not_found }}$ which refers to unsuccessful paging; it is the maximum time that is waited for a response from the paged MT after the broadcasting of a paging message.

\begin{tabular}{|l|l|l|}
\hline & $\begin{array}{c}\text { MT is in its registered } \\
\text { PA }\end{array}$ & $\begin{array}{c}\text { MT is elsewhere in } \\
\text { the LA }\end{array}$ \\
\hline Paging delay & $d_{\text {found }}$ & $d_{\text {found }}+d_{\text {not found }}$ \\
\hline Paging needed & only in one PA & in entire LA \\
\hline
\end{tabular}

Table 1: Characteristics of paging scenario

In [11] an estimation of the probability that the MT is still in its registered PA is given. This probability is defined as $p_{i n}$, and can be derived based on the model developed in [9].

Using this probability $p_{i n}$ we are able to calculate the mean number of paging areas to be paged per incoming call to a MT $\left(\overline{n_{\text {paging }}}\right)$, and the mean paging delay $\left(\overline{d_{\text {paging }}}\right)$. In the formula $n$ represents the number of PAs in a LA.

$$
\begin{gathered}
\overline{n_{\text {paging }}}=p_{\text {in }} \cdot 1+\left(1-p_{\text {in }}\right) \cdot n \\
\overline{d_{\text {paging }}}-d_{\text {found }}+\left(1-p_{\text {in }}\right) \cdot d_{\text {not_found }}
\end{gathered}
$$

The features of this scenario can be summarized as follows: Location update trigger: Paging:

crossing of LA border two-step; first in the registered PA, next in rest of LA

Paging Areas: Location Areas: LA is divided into several PAs fixed

\section{Timed Location Updating Scenario}

In this scenario the paging and location area terminology as defined in the previous scenario can only be applied with some specific observations. The UMTS coverage area is divided into a number of areas, each identifiable by their unique ID number. The areas serve both as a reference for the location updating procedure, and for paging. Further on we will refer to these areas as PAs. The term LA will not be used.

The MTs perform a location update based on a timer. The timer starts at the moment an MT leaves its registered PA. After a predefined number of seconds the timer will elapse, and at that moment the MT will perform a location update. The MT will not perform a location update if it has returned to the same PA meanwhile.

Paging has to be done in a multiple-step fashion; first in the registered PA, next in the PAs surrounding the registered PA, next in the PAs surrounding those PAs, etc. This will continue until the MT is found, or until the maximum number of paging steps has been reached. Since the speed of a MT is limited (e.g., max. 50 $\mathrm{km} / \mathrm{h}$ in urban areas), such a maximum exists. In order to avoid an excessively high number of paging steps (and thus an unacceptable large connection setup delay), the size of the PAs has to be chosen with care. Note that in the real world the PA layout may not be as ideal as shown in Figure 2.

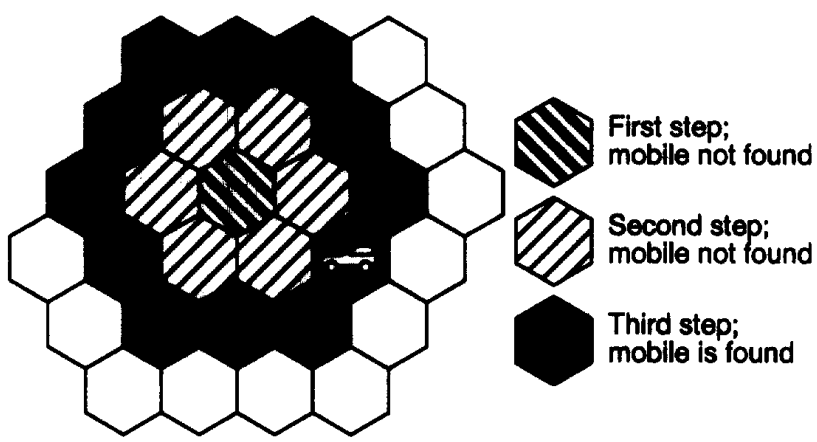

Figure 2: Example of $n$-step paging procedure

The characteristics of this scenario can be summarized as follows: Location Update trigger: time-out (starting at PA border crossPaging: ing, only if PA is changed) $n$-step; first in PA in which the last contact between the paged MT and the fixed network occurred, next in surrounding bands of PAs

Paging Areas: fixed

\section{UMTS Scenario Definition}

This section describes the input for the analysis. In order to do the evaluation, it is necessary to model the expected UMTS user behaviour. This behaviour can be seen as consisting of two separate, independent parts: (1) the mobility behaviour, modelled by the mobility model in sub-section 4.1 , which describes the way a user moves through the system, and (2) the traffic behaviour, modelled by the traffic model in sub-section 4.2 , which describes the call behaviour. Furthermore it is necessary to model the layout of paging and location areas, which is done by the network model in subsection 4.3 . 


\subsection{Mobility Model}

As stated before, the aim of this study is not to provide very accurate predictions for actual user behaviour, but to provide rough figures for comparison of design alternatives. Therefore the mobility model used will be quite simple compared to the mobility models derived in chapters 2 and 3 of [13]. The model that we are using is based on [4][6] and [11].

The behaviour of the users is modelled by splitting the user population into two groups; the stationary and the moving users. In the mobility model described in [11] the moving users are split into more classes, such as car passenger and pedestrian. We will refrain from this detailed split, and look at the user behaviour for one average speed. An extension of this model may also take into account this more detailed split into user classes. In the following we will look in detail at the behaviour of the moving users.

\section{Moving users}

In [6] a formula is introduced to estimate the rate of user border crossings for a given circular area. We assume that the density of users is uniform throughout this area, and that the direction of motion with respect to the border is uniform on $[0,2 \pi]$. Let $\rho$ be the density of moving users in the area, $v$ be a users average speed, and $l$ be the area border length. Then, the rate of border crossings per hour $(C R)$ out of this area is given by:

$$
C R=\frac{\rho v l}{\pi}
$$

This formula is used as an estimation for the border crossing rate of non-circular areas, by looking at a circular area with the same size as the area we are interested in. For hexagonal shaped areas this is a reasonable approximation. The value of $l$ is estimated by using the formulae for the area and circumference of a circle with size $a$ :

$$
l=2 \cdot \sqrt{\pi \cdot a}
$$

so that formula (3) becomes:

$$
C R=2 \frac{\rho v \sqrt{a}}{\sqrt{\pi}}=1.128 \cdot \rho v \sqrt{a}
$$

The following step is to model the behaviour of a single moving user with the assumption of a negative exponential residence time in an area. In order to obtain the area crossing rate for a single moving user ( $c r$ ), we divide (5) by the total number of moving users in that area $(\rho a)$, resulting in:

$$
c r=2 \frac{v}{\sqrt{\pi a}}=1.128 \cdot v / \sqrt{a}
$$

\section{Fixed LA scenario}

Applying formula (6) to a LA containing $n$ equally large PAs, each with size $a_{P A}$, we obtain for the border crossing rate $\gamma$ for one moving user out of a PA:

$$
\gamma=2 \frac{v}{\sqrt{\pi a_{P A}}}
$$

and the border crossing rate $\mu$ for one moving user out of a LA:

$$
\mu=2 \frac{v}{\sqrt{\pi n a_{P A}}}=\gamma / \sqrt{n}
$$

A user that crosses the LA border will, at the same moment, also cross a PA border. If we want to obtain the rate $\lambda$ for PA crossings for which the user stays in the same $L A$, we must subtract $\mu$ from $\gamma$. The following is obtained:

$$
\lambda=\gamma-\mu=\gamma \frac{\sqrt{n}-1}{\sqrt{n}}
$$

\section{Timed location update scenario}

Formula (7) can be used to estimate the border crossing rate of a moving user. In this scenario we are interested in the distance that a user is away from its registered PA. A worst-case assumption is that the user will always move away from that PA. This assumption will be used in the model defined in sub-section 5.2.

\section{Assumptions}

The following figures are expected to give a reasonable representation of the average behaviour of the future UMTS users:

- the probability that a MT is moving ( $\left.p_{\text {moving }}\right)$ is $20 \%$,

- the average speed of a moving MT $(v)$ is $20 \mathrm{~km} / \mathrm{h}$.

\subsection{Traffic Model}

The traffic behaviour of the UMTS users can be defined in a similar way as it is already defined in existing fixed networks. Again, for reasons of simplicity, the traffic model used will be quite simple. A total average call rate of 3 calls per hour per MT is assumed, corresponding to the expected traffic load due to telephone calls in the UMTS network [12]. Half of all calls are incoming, and half are outgoing. The interarrival time of the calls is assumed to have a negative exponential distribution. Because of the fact that in our forthcoming evaluation the call length and type is of minimal importance, it will not be defined.

Hence, the following traffic model is used:

- the arrival rate of incoming calls per MT is $1 / 2400$ per second, - the arrival rate of outgoing calls per MT is $1 / 2400$ per second.

\subsection{Network Model}

This section defines a simple network model containing the layout of paging and location areas. We assume that all PAs have the same size (and similar shape), and that a LA exists out of a fixed number of PAs. The size of PAs and the number of PAs per LA are variable, so that we can optimise the performance of the scenario under investigation. For the density of the UMTS users $(\rho)$ we assume $\mathbf{8 0 0}$ users per square $\mathrm{km}$. This figure is not important in an absolute sense; all results can be scaled according to the actual user density, so the relative performance of the scenarios is independent of this figure.

\section{Evaluation Model}

The scenarios we will use are described in Sections 2 and 3 . The UMTS scenario definition of Section 4 will be used as input for this evaluation. 


\subsection{Fixed Location Area Scenario \\ 5.1.1. Location Updates}

Stationary MTs do not produce location updates, so we see immediately that the average rate of location updates produced per MT $\left(\overline{r_{L U}}\right)$ can be defined as:

$$
\overline{r_{L U}}=p_{\text {moving }} \cdot \mu=p_{\text {moving }} \cdot \gamma / \sqrt{n}
$$

\subsubsection{Paging}

In order to calculate the number of paging messages for scenario 1 it is necessary to develop a model for the state of a MT, in which several states of the terminal leading to different numbers of paging messages needed for incoming calls, are distinguished. We define two states:

- state 0: the MT is still in its registered PA, in this case the network only has to page in one PA in case of an incoming call,

- state 1: the MT is not in its registered PA, so all PAs in the LA must be paged if there is an incoming call.

Excluding special interactions, such as the switching-on of a terminal, the following events cause a MT to move from one state to the other:

- state 0 to state 1: a movement of the MT, in which it crosses a PA border (but not a LA border)

- state 1 to state 0: either the occurrence of an incoming or outgoing call, or a movement of the MT, in which it crosses a LA border (so a location update is performed).

Figure 3 shows a simple Markovian model that models this behaviour:

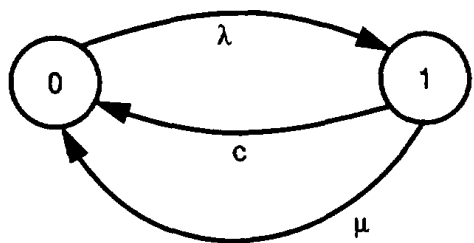

$\lambda=$ rate of movement into another PA (same LA)

$\mu=$ rate of movement into another $L A$

$\mathrm{C}=$ rate of incoming plus outgoing calls

Figure 3: Markov model of MT states, fixed LA scenario

The movement rate of a MT out of a PA into another PA of the same LA is $\lambda$, and the movement rate of a MT out of the current LA into another LA is $\mu$. Calls arrive with an average inter-arrival time of $1 / c$. The fact that during a call no paging is needed (in case of another incoming call), is not modelled. If we assume the length of the calls to be small compared to the residence time in a PA, this is acceptable.

For stationary MTs both $\lambda$ and $\mu$ are equal to zero. In that case the probability for a MT of being in state 0 or 1 can only be analysed in a transient way, given the stationary probabilities at the end of a movement as a starting point. Because of the inability to calculate the input for this approach, and being aware of the inherent inaccuracy in the other input figures, we will assume that stationary MTs are always in state 0 , i.e., they have to be paged in only one PA.

By solving for the stationary distribution of this Markov model one can derive that [14]:

$$
\overline{n_{\text {paging }}}=p_{\text {moving }} \cdot\left(\frac{c+\gamma \cdot v}{\gamma+c}\right)+p_{\text {non-moving }} \cdot 1
$$

in which $v=n-\sqrt{n}+1 / \sqrt{n}$.

\subsubsection{Terminal Paging Delay}

The terminal paging delay ( $\overline{d_{\text {paging }}}$ ) is defined in Equation (2). It can be shown that for this scenario [14]:

$$
\overline{d_{\text {paging }}}=p_{\text {moving }} \cdot \frac{\sqrt{n}-1}{\sqrt{n}} \cdot \frac{\gamma}{\gamma+c} \cdot d_{\text {not_found }}+d_{\text {found }}
$$

\subsection{Timed Location Updating Scenario}

In order to calculate a mean value for the number of PAs in which must be paged in case of an incoming call, we will have to define a larger number of states than in the fixed LA scenario. Since the location updates are performed only after the expiry of a timer started at the crossing of a PA border, a MT may have moved through several PAs, at the moment an incoming call arrives. The paging is done in a multiple-step fashion as shown in Figure 2. The state of a MT will now refer to the distance of the MT to its registered PA. The states are numbered upwards starting at 0 . State 0 is when the MT is still in its registered PA, state $n$ means that the MT is at $n$ PAs distance from that PA. A state-transition model is depicted in Figure 4.

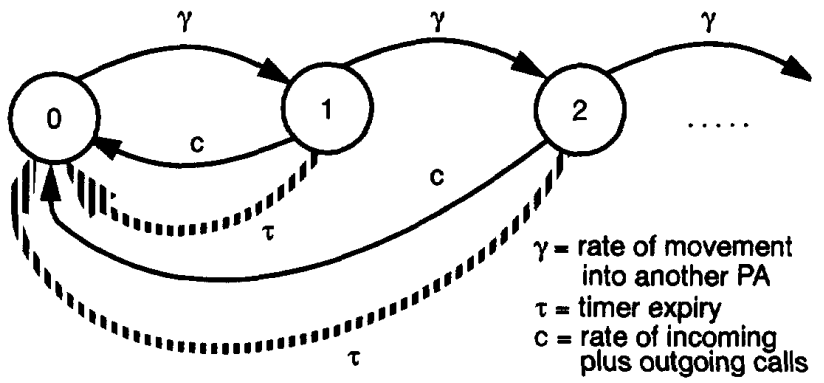

Figure 4: State-transition model of MT states, timed location update scenario

The timer starts at the moment of the transition from state 0 to state 1 . The time-out event is modelled by the transition $\tau$.

\subsubsection{Location Updates}

For moving users the average number of location updates can be calculated by determination of the mean rate of $\tau$-transitions. Stationary users may have the timer still running, and do one final location update on the expiry of their timer. Due to the difficulty in estimating this, we will assume that stationary users will not generate location updates. The average location update rate per MT is then:

$$
\begin{array}{r}
\overline{r_{L U}}=p_{\text {moving }} \cdot \tau \\
\text { 5.2.2. Paging }
\end{array}
$$

Stationary users of which the timer has not yet expired, must be paged in several PAs. Since it is difficult to provide figures, we will assume that stationary users have already done their final location update, so only 1 paging message will be needed per incoming call. 
For moving users the mean paging rate can be determined by calculation of the steady-state probabilities of the model of Figure 4. Since we assume the call arrival process to be Poisson distributed, this steady state probability will be experienced by arriving calls. The average number of paging messages needed for an incoming call is:

$$
\overline{n_{\text {paging }}}=p_{\text {moving }} \cdot\left(\sum_{i=0}^{\infty} n(i) \cdot p_{i}\right)+p_{\text {stationary }} \cdot 1
$$

In which $n(i)$ denotes the number of PAs that need to be paged in case the MT has moved $i$ PAs away, and is defined as:

$$
n(i)=1+3 \cdot i \cdot(i+1)
$$

\subsubsection{Terminal Paging Delay}

For this scenario the average terminal paging delay equals [14]:

$$
\overline{d_{\text {paging }}}=d_{\text {found }}+p_{\text {moving }} \cdot d_{\text {not_found }} \cdot \sum_{i=1}^{\infty} i \cdot p_{i}
$$

\subsubsection{Estimation of steady-state probabilities}

In order to get the required performance parameters for moving MTs, it is necessary to obtain values for $\tau$ and all $p_{i}$ probabilities. For this means the UltraSAN tool [7] is used. The state-transition model of Figure 4 is transformed into a Stochastic Activity Network (SAN), shown in Figure 5.

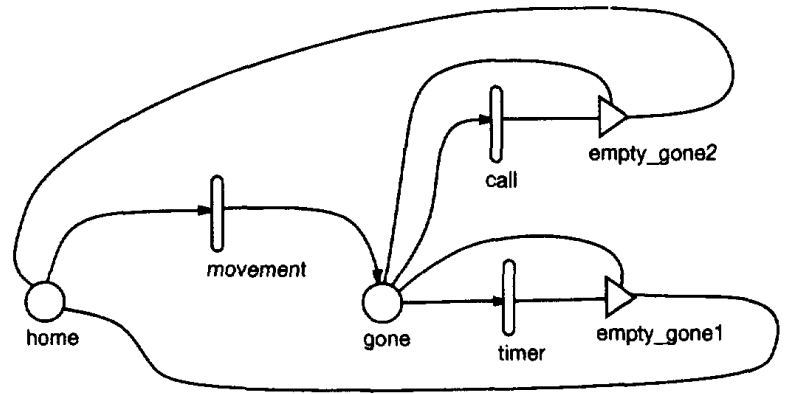

Figure 5: UltraSAN model of MT states, timed location update scenario

The SAN contains two places, home and gone. The state of the original state-transition model is represented by the number of tokens in place gone. The infinite chain is now truncated to a finite length, represented by the initial number of tokens in place home. As a first try we took 20 tokens. Movement of the MT is modelled by the timed transition movement, having a negative exponential distribution $\gamma$, as defined in our mobility model. Calls to and from the MT are modelled by the timed transition call. The deterministic timer is modelled by the timed transition timer, with an Erlang$n$ distribution ${ }^{1}$. The output gates empty_gonel and empty_gone2 take all tokens out of place gone, and reset place home to its initial state.

The tool is able to calculate the steady-state distribution of the tokens, and to calculate the firing rate of transitions. The later is used to obtain the value for $\tau$. 1. In which $n$ is taken large enough in order to get sufficiently accurate
results.

\section{Results of evaluation}

The scenarios are compared on three aspects:

- the mean paging rate per paging area,

- the average location updating rate generated by a MT, and,

- the mean terminal paging delay in case of an incoming call.

\subsection{Mean Paging rate per Paging Area}

Figure 6 shows the mean paging rate per PA. The left part of the figure shows the graph for the fixed LA scenario. The mean paging rate is depicted for LAs consisting of $1,7,19$ or 37 PAs. The right part of the figure shows the mean paging rate for the timed location updating scenario, for time-out values of $200,400,800$ and 1600 seconds.
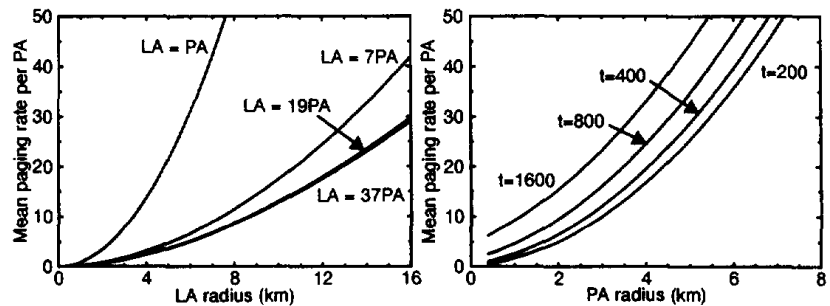

Figure 6: Mean paging rate per PA for both scenarios

We observe that the paging rate per area increases as the area size (and so the number of users in the area) increases, which was to be expected. In the left part of the figure we observe that the division of the LA into PAs is beneficial. Our research indicated that there is an optimal division; if we let the number of PAs approach to infinity, the paging load is higher than the optimum shown in the graph (for 37 PAs per LA). In the right part of the figure we see that as the timer value increases, the number of paging messages also increases, which is easy to explain because in that case the average distance of a MT from its registered PA will also increase (so more paging steps will be needed). The advantage of this is that we are able to, without making changes in the physical network, adjust the number of paging messages by adjusting the timer value.

\subsection{Impact on the DDB Load}

The impact of the scenarios on the load of the DDB is defined by the rate of location updates. Figure 7 shows the relation between the location update rate per user and either the LA or PA size. The total location update rate on the DDB can be calculated by multiplication of the location update rate per user and the total number of users covered by the DDB.
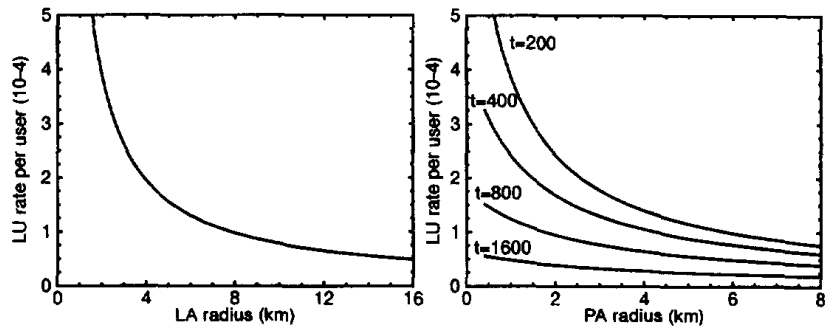

Figure 7: Mean location update rate per MT for both scenarios 
We observe that in both cases the location update rate is inverse proportional to the LA/PA size. In case of the timed location update scenario the location update rate is also dependent on the size of the timer: by increasing the timer value we are able to decrease the location update rate. Again the advantage of this is that we are able to adjust the generated location update rate by adjusting the timer value.

\subsection{Impact on the Call Setup Delay due to Paging}

In order to look at the call setup delay due to paging we assume that both $d_{\text {found }}$ and $d_{\text {not found }}$ equal $500 \mathrm{~ms}$. The left part of Figure 8 shows the mean $d$ paging for the fixed LA scenario. The right part shows the mean $d_{\text {paging }}$ for the timed location updating scenario.

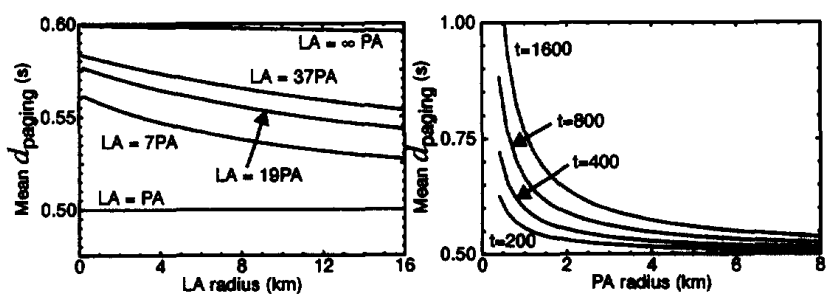

Figure 8: Mean $d_{\text {paging }}$ for both scenarios

For the fixed LA scenario the upper limit for $d_{\text {paging }}$ is shown by

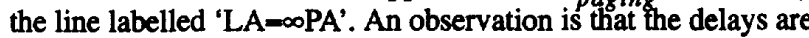
generally much higher for the timed location update scenario. However, by careful adjustment of the PA radius and the timer value, we are able to keep the delay below an acceptable value. Note that the actual values for $d_{\text {found }}, d_{\text {not found }}$ and the maximum accepted call setup delay also play an important role in this determination.

\section{Conclusions and Future Research}

Combining Figures 6 and 7, we see that by using the new, timed location update scenario, with a careful choice of the timer value we are able to outperform the fixed LA scenario. The added advantage is that we are able to balance the load between paging and location updates, by only changing the timer value, which can even be done in an operational system, without interruption of service provisioning. Another advantage is the fact that we are now able to optimise the paging and location updates on a per-terminal base, by adjusting the timer in the terminal. It is then possible to avoid the overhead in location updates that we have for terminals with a low incoming call rate and moving at high speeds. This subject is for further study. In the fixed LA scenario there is a need for an extensive LA planning, in order to optimise the number of location updates in a real geographical area like a large city [3]. By using the proposed timed location update scenario this kind of planning is unnecessary, because only one parameter (the average size of the PAs) must be set. The final advantage of the new scenario is the fact that now location updates are spread over all radio cells in the network. This is opposed to the fixed LA scenario, in which all messages related to location updates are concentrated in the radio cells located at the LA borders, thus creating a higher load on the radio channels in these cells.

Subjects for further research are:
- Using a mobility model that takes into account a mix of average speeds for the users by looking at more user classes like pedestrian and car passenger.

- Investigation into the peak behaviour of the performance parameters. In particular this is interesting for the terminal paging delay.

- Looking at variants of the timed location update scenario:

- investigation into an adaptive setting of the timers in the MTs, and

- impact of limiting the distance of MTs to do a location update.

\section{References}

[1] B. Samadi \& W.S. Wong: Optimization Techniques for Location Area Partitioning. In: Proceedings 8th ITC Specialist Seminar on Universal Personal Telecommunication. San Margarita, Genova, Italy. October 12-14, 1992. pp. 141-147

[2] B.R. Badrinath, T. Imielinski \& A. Virmani: Locating Strategies for Personal Communication Networks. In: Workshop on Networking of Personal Communications Applications. December, 1992

[3] G.L. Lyberopoulos, J.G. Markoulidakis \& E.D. Sykas: Intelligent paging strategies in future mobile telecommunication networks. In: Proceedings COMCON 4. Rhodos, Greece. 14-18 June 1993.

[4] E. Alonso, K.S. Meier-Hellstern \& G.P. Pollini: "Influence of Cell Geometry on Handover and Registration Rates in Cellular and Universal Personal Telecommunications Networks". In: Proceedings 8th ITC Specialist Seminar on Universal Personal Telecommunications. Santa Margarita, Genova, Italy. October 12-14, 1992. pp. 261-270.

[5] S. Okasaka, S. Onoe, S. Yasuda \& A. Maebara: "A new location Updating Method for digital cellular systems" In: Proceedings 41 st VTS Conference, St. Louis, Missouri. May 1991.

[6] G. Morales-Andres \& M. Villen-Altamirano: An approach to modelling subscribers mobility in cellular radio networks. In: Forum Telecom 87. Geneva, 1987.

[7] J. Couvillion, R. Freire, R. Johnson, W.D. Obal, A. Qureshi, M. Rai, W.H. Sanders \& J.E. Tvedt: "Performability Modelling with UltraSAN" IEEE Software, vol. 8, no. 5, Sept. 1991, pages 69-80.

[8] H. de Boer, M. Meijer \& E. Buitenwerf: Network aspects for the third generation mobiles. In: Proceedings GLOBECOM 1991.pp. 42.5.1-42.5.6

[9] Final Report on Fixed Network aspects in UMTS. CEC deliverable 43/RNL/FN12/DS/A/067/b1, issue $2.0,13$ December 1991.

[10] Location areas, paging areas and the Network Architecture. CEC deliverable R2066/PTTNL/MF1/DS/P/001/b1. 1 May 1992.

[11] Mobility model for UMTS (final). CEC deliverable R2066/ SESA/GA2/DS/P/015/b2, November 1993.

[12] Results of Traffic Modelling for UMTS. CEC deliverable R2066/SESA/GA2/DS/P/030/b1, July 1993.

[13] Signalling Traffic Requirements for UMTS. CEC deliverable R2066/SESA/GA2/DS/P/054/b1, February 1994.

[14] F.V. Baumann: An Evaluation of Location Management Procedures for UMTS. Memoranda Informatica 9436. June 1994. University of Twente, Dept. of CS. 